\title{
Navigating the negative-mode proteome
}

A negative-mode mass spectrometry approach characterizes intractable parts of the proteome.

The impressive advances in the proteomics field in the past two decades were spurred in no small part by electrospray ionization, a gentle technique used to produce gas-phase peptide ions for tandem mass spectrometry (MS/MS) analysis. The vast majority of studies have relied on adding a positive charge to ionize peptides. However, acidic peptides will not readily accept a positive charge, making them difficult to ionize. Many posttranslationally modified peptides are acidic, making large classes of modified peptides resistant to characterization.

Applying a negative charge to acidic peptides also results in their ionization, but this approach produces MS/MS spectra that are too complex to interpret using traditional fragmentation methods. However, there are work-arounds. In particular, negative electron-transfer dissociation (NETD) uses electron-driven fragmentation to enable sequencing of peptides ionized under negative electrospray conditions.

In recent work, Joshua Coon of the University of Wisconsin-Madison and colleagues implemented activated ion negative electron-transfer dissociation (AI-NETD) on a large scale for the first time. In AI-NETD, peptide ions are irradiated with infrared light concurrently with the NETD reaction, which improves peptide fragmentation over that achieved with NETD alone.

Working in yeast, Coon and colleagues first tested just how much proteome coverage they could achieve in a single-shot AI-NETD experiment using a single protease, trypsin, to break up the proteins into peptides. They detected 7,601 unique peptides from 1,106 proteins. Next, they tested a combination of five different proteases, which has been shown to improve proteomic coverage compared with using trypsin alone. They detected 1,359 proteins (and more than 21,000 peptides) by AI-NETD. Last, they applied extensive low-pH reversed-phase fractionation prior to AI-NETD. This provided a major boost, yielding 3,730 protein identifications, representing $83 \%$ of the expressed yeast proteome, with an average sequence coverage of $44 \%$.

The Coon team's AI-NETD peptide identifications overlapped by just $18 \%$ with those from a state-of-the-art positive-mode analysis, and 272 proteins were detected only with AI-NETD. AI-NETD may find its place as a powerful complement to current proteomics approaches.

\section{Allison Doerr}

\section{RESEARCH PAPERS}

Riley, N.M. et al. The negative mode proteome with activated ion negative electron transfer dissociation. Mol. Cell. Proteomics doi:10.1074/mcp.M115.049726 (20 July 2015). 